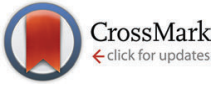

Cite this: J. Mater. Chem. B, 2016, 4, 4017

Received 23rd February 2016, Accepted 5th May 2016

DOI: $10.1039 / c 6 t b 00458$

www.rsc.org/MaterialsB

\title{
A facile construction of gradient micro-patterned OCP coatings on medical titanium for high throughput evaluation of biocompatibility $\dagger$
}

\author{
Ran Song, ${ }^{a}$ Jianhe Liang, ${ }^{a}$ Longxiang Lin, ${ }^{a}$ Yanmei Zhang, ${ }^{\text {bc }}$ Yun Yang ${ }^{\text {bc }}$ and \\ Changjian Lin*abc
}

\begin{abstract}
Base on a superhydrophobic-superhydrophilic micro-patterned template, a facile construction of gradient micro-patterned octacalcium phosphate (OCP) coatings on titanium has been firstly developed for highthroughput evaluation of biocompatibility. The gradient OCP coatings with tunable crystal morphologies involving scattered-flower-like, scattered-flower-ribbon-like, short-ribbon-like and long-ribbon-like were fabricated in different micro-units on the same surface. The significant difference of mineralization behavior of the gradient OCP coatings in the micro-patterns was observed visually and efficiently. In vitro cultures of MC3T3-E1 cells showed that the number and morphology of cells selectively adhered on the micro-units of gradient structure of OCP coatings were distinctly different, indicating that the cells are sensitive to the different structures of OCP coatings on medical titanium. The gradient micropatterned construction is potentially a powerful method for not only high-throughput screening of the biocompatibility of various biomaterials, but also efficient development of advanced biomaterials by controlling cell immobilization and inducing cell response.
\end{abstract}

\section{Introduction}

Surface chemistry, morphology and wettability play an important role in cell adhesion, proliferation and biological response..$^{1-3}$ Avoiding the disadvantages of conventional research methods, such as tedious processes, consuming time, large amount of samples, cost and accidental error, micro-patterned surfaces behave with an outstanding function in high-throughput investigation of complex cell life behavior. ${ }^{4-11}$ Superhydrophobicity with a water contact angle (CA) above $150^{\circ}$ and superhydrophilicity with a CA below $5^{\circ}$ are the two extreme cases of wettability and have attracted great interest due to their importance in fundamental research and practical application. ${ }^{12,13}$ The differences of wettability can be used to engineer micro-templates with various sized micro-units of domains, which as a unique prospective technique has been widely applied in cell growth, ${ }^{14,15}$ fluid microchips, ${ }^{16}$ micro-reactors, ${ }^{17,18}$ and sensor arrays. ${ }^{19}$ Among various applications, the micro-patterning of living cells is attracting more and more attention because it can be used in

\footnotetext{
${ }^{a}$ State Key Laboratory for Physical Chemistry of Solid Surfaces, and Department of Chemistry, College of Chemistry and Chemical Engineering, Xiamen University, Xiamen 361005, China. E-mail: cjlin@xmu.edu.cn

${ }^{b}$ Beijing Medical Implant Engineering Research Center, Beijing 100082, China ${ }^{c}$ Beijing Engineering Laboratory of Functional Medical Materials and Devices, Beijing 100082, China

$\dagger$ Electronic supplementary information (ESI) available. See DOI: 10.1039/c6tb00458j
}

a broad range of research such as bioassays, ${ }^{20,21} \mathrm{drugs},{ }^{22}$ tissue engineering, ${ }^{23}$ and many fundamental studies of interactions between cells and materials. ${ }^{24-28}$ Recent progress in micropatterning techniques has enabled the control of most of the crucial parameters of the cell microenvironment. Scopelliti et al. introduced novel methods for quantitative high-throughput characterization of protein-surface interactions, and indicated that the adsorption of proteins depended closely on the surface nanostructure, and the relevant morphological parameter regulating the protein adsorption was nanometric pore shape. ${ }^{29}$ Ito et al. investigated cells on a photo-immobilized protein micro-array and found that the adhesion behavior of cells relied on the kind of immobilized proteins and the kind of cells. ${ }^{30}$ Thompson et al. developed a method of controlling Schwann cell placement and orientation in vitro by using microlithographically patterned laminin substrates..$^{31}$ Ye et al. reported an effective technique for controlling the shape and spreading of cells by a poly micro-pattern, and square-shaped mouse osteoblast MC3T3-E1 cells were obtained in micro-well arrays. ${ }^{7}$ Tang et al. investigated both osteogenic and adipogenic differentiations of mesenchymal stem cells on a micro-patterned oil polyethylene glycol hydrogel, which were fairly linearly related to the extent of contact characterized by coordination number. ${ }^{32}$ Shi et al. constructed a polydopamine (PDA)-coated parafilm with grooved micro-patterns to induce the osteogenic differentiation of stem cells. They found that the adipose-derived 
mesenchymal stem cells cultured on the PDA-coated parafilm exhibited significantly higher osteogenic commitment in response to mechanical and spatial cues compared to the ones without stretch. ${ }^{33}$ Yao et al. prepared an anion-exchangeable MgAl layered double hydroxide micro-pattern to act as both bioadhesive region for selective cell adhesion and nanocarrier for drug molecules to regulate cell behaviors. ${ }^{34}$ Engineered micro-patterns are able to provide a micrometer-scale, 3-dimensional and complex microenvironment for individual cells or for multicellular arrangements. The relationship of cell behaviors and micro-patterned morphology, composition and structure can be detected and evaluated in a high-throughput way.

Titanium implants are extensively used for biomedical devices because of their favorable biocompatibility and mechanical properties, while osseointegration of them is limited since they are generally encapsulated by fibrous tissue after implantation. ${ }^{35-38}$ Therefore, bioactive calcium phosphate $(\mathrm{CaP})$ coatings or/and other chemical or biochemical modifications are frequently used to improve the bone integration properties. ${ }^{39}$ Among them, octacalcium phosphate $\left(\mathrm{OCP}, \mathrm{Ca}_{8} \mathrm{H}_{2}\left(\mathrm{PO}_{4}\right)_{6} \cdot 5 \mathrm{H}_{2} \mathrm{O}\right)$ is of particular interest in tissue mineralization biochemistry, ${ }^{40,41}$ since it has been reported to be a direct precursor phase during the biomineralization process of bone and teeth. ${ }^{42,43}$ Previous work of our group demonstrated that modification by a thin OCP layer enhanced bioactivity of roughened medical titanium. ${ }^{44}$ Although OCP coatings with biocompatible structure have become a popular field, very few researches focusing on the relationship between different crystal morphologies of OCP coatings with various micro-patterns and their bioactivity have been reported. ${ }^{45,46}$ In this work, we aimed to construct gradient OCP coatings with tunable crystal morphologies in various micro-patterns on the same medical titanium surface to investigate the dependence of bioactivity and biocompatibility of OCP coatings on their crystal morphologies in a high-throughput way.

Plentiful methods for micro-patterned surfaces have been developed, such as photolithography, ${ }^{47-50}$ ink-jet printing, ${ }^{51}$ soft lithography ${ }^{52-55}$ and micro-contact printing. ${ }^{56-61}$ However, most of the conventional methods and new techniques can be only used for microfabrication on glass, silicon wafers or specific material. The materials chosen to be filled in the micro-patterns are also specific, such as proteins, polymers, hydrogels and drug molecules, and costly equipment and rigorous fabrication conditions are generally required for micro-pattern fabrication. A facile, tunable and cheap microfabrication technique which is able to produce gradient micro-patterns on various functional materials is urgently needed. $\mathrm{TiO}_{2}$ nanotube array (NTA) films fabricated by electrochemical anodizing have been widely studied in various applications, such as biomaterials, ${ }^{62,63}$ optoelectronics, ${ }^{64}$ photocatalysts ${ }^{65,66}$ and solar cells. ${ }^{67,68}$ Based on the precisely controllable ability of superhydrophilicsuperhydrophobic properties of $\mathrm{TiO}_{2}$ NTA films, by surface assembling and photocatalysis, ${ }^{69-77}$ here we demonstrated a unique and advanced approach to fabricate gradient micro-patterned OCP coatings with biocompatible structure on medical titanium, which can be efficient and powerful to study biocompatibility and cell-material interactions. As a micro-patterned material, the gradient micro-patterned OCP coatings have unique gradient function that can be used to perform more effective highthroughput investigation, avoiding the disadvantages of conventional research methods, such as tedious processes, consuming time, large amount of samples, cost and accidental error.

\section{Experimental}

\section{1. $\mathrm{TiO}_{2}$ micro-patterned templates}

Firstly, the $\mathrm{TiO}_{2}$ NTAs were prepared by electrochemical anodizing on titanium sheets (purity 99.5\%) in $0.5 \mathrm{wt} \% \mathrm{HF}$ electrolyte with Pt counter electrode under $20 \mathrm{~V}$ for $20 \mathrm{~min}$. Then molecular assembling was conducted on the $\mathrm{TiO}_{2}$ NTAs in a methanolic solution with 1 vol\% $1 H, 2 H, 3 H, 4 H$-perfluorooctyltriethoxysilane (PTES, Degussa) for $1 \mathrm{~h}$ and then heated at $140{ }^{\circ} \mathrm{C}$ for $1 \mathrm{~h}$ to form a superhydrophobic film. Then, the sample surface was exposed to ultraviolet irradiation through a photomask with various sizes of micro-patterns for an appropriate period of time to selectively cleave the fluoroalkyl chain. Finally, the $\mathrm{TiO}_{2}$ micro-templates with superhydrophobic-superhydrophilic patterns were prepared. ${ }^{78}$

\subsection{Gradient electro-depositing of OCP}

The as-prepared superhydrophobic-superhydrophilic micro-patterns were used as a template for electrodepositing OCP on the superhydrophilic units only in an aqueous electrolyte containing $0.042 \mathrm{~mol} \mathrm{~L}^{-1} \mathrm{Ca}\left(\mathrm{NO}_{3}\right)_{2}$ and $0.025 \mathrm{~mol} \mathrm{~L}^{-1} \mathrm{NH}_{4} \mathrm{H}_{2} \mathrm{PO}_{4}$ at $\mathrm{pH} 4.5$ under galvanostatic mode to form OCP micro-patterns on titanium surface. During the OCP electrodeposition, a pull-stop-pull method was used to control the sample moving out of the solution at a certain rate to enable gradient OCP coatings deposited on the different sized micro-patterns.

\subsection{Simulated body fluid immersion test}

The bioactivity of the as-prepared gradient micro-patterned OCP coatings was examined from the biomineralization in simulated body fluid (SBF). Samples with gradient OCP micro-patterns were immersed into SBF, which mimicked the composition of the inorganic part of human blood plasma. The SBF was prepared based on Kokubo's formulation by dissolving $8.035 \mathrm{~g} \mathrm{~L}^{-1} \mathrm{NaCl}, 0.225 \mathrm{~g} \mathrm{~L}^{-1}$ $\mathrm{KCl}, 0.355 \mathrm{~g} \mathrm{~L}^{-1} \mathrm{NaHCO}_{3}, 0.311 \mathrm{~g} \mathrm{~L}^{-1} \mathrm{MgCl}_{2} \cdot 6 \mathrm{H}_{2} \mathrm{O}, 0.292 \mathrm{~g} \mathrm{~L}^{-1}$ $\mathrm{CaCl}_{2}, 0.231 \mathrm{~g} \mathrm{~L}^{-1} \mathrm{KH}_{2} \mathrm{PO}_{4} \cdot 3 \mathrm{H}_{2} \mathrm{O}$ and $0.072 \mathrm{~g} \mathrm{~L}^{-1} \mathrm{Na}_{2} \mathrm{SO}_{4}$ into double distilled water buffered with tris-hydroxymethylaminomethane to $\mathrm{pH}=7.3$ at $37{ }^{\circ} \mathrm{C}$, and $\mathrm{NaN}_{3}$ was added to inhibit the growth of bacteria. The samples were exposed to SBF under static conditions for 3 days for evaluating the transformation of OCP to hydroxyapatite (HA). The samples were thoroughly rinsed with double distilled water after immersion, and then air-dried for further examinations.

\subsection{Surface characterization}

The surface morphologies and chemical compositions of the gradient micro-patterned OCP coatings and the mineralization products were characterized by a field emission scanning electron microscope (FE-SEM, Hitachi S4800, Japan) equipped with energy-dispersive X-ray spectroscopy (EDS, Inca system, 
Oxford Instruments, UK) capability and an electron probe micro-analyzer (EPMA, JXA 8100, JEOL, Japan). The crystalline phases of the samples were identified using an X-ray diffractometer (XRD, Rigaku Ultima IV, Japan) with $\mathrm{Cu}-\mathrm{Ka}$ radiation source at $35 \mathrm{kV}$ and $15 \mathrm{~mA}$. Three-dimensional profile images on surfaces were measured using a 3D Optical Surface Metrology System (DCM 3D, Leica Microsystems, Germany).

\subsection{Cell culture}

Mouse MC3T3-E1 preosteoblastic cells were used for all biological assays. The MC3T3-E1 cells were cultured in medium containing alpha-minimum essential medium ( $\alpha$-MEM) supplemented with $10 \%$ FBS, 100 units per $\mathrm{mL}$ penicillin, and $100 \mu \mathrm{g} \mathrm{mL}$ streptomycin and maintained under a humidified atmosphere with $5 \% \mathrm{CO}_{2}$ at $37^{\circ} \mathrm{C}$. The cells were detached from the culture dish when reaching $90 \%$ confluence with $0.25 \%$ trypsin, centrifuged at $1000 \mathrm{rpm}$ for $5 \mathrm{~min}$, and resuspended in fresh culture medium. Four groups of samples (gradient micropatterned OCP coatings, circle with diameter of $100 \mu \mathrm{m}$ and gap length of $100 \mu \mathrm{m}$ ) were sterilized by soaking in $75 \%$ alcohol for $1 \mathrm{~h}$, air-dried and wetted with phosphate buffer saline (PBS). Then the samples were placed into a 6-well plate and seeded with cells at a density of $5 \times 10^{4}$ cells per $\mathrm{cm}^{2}$.

\subsection{Cell adhesion and morphology}

After incubating for $6 \mathrm{~h}$ and $24 \mathrm{~h}$, all groups of samples were rinsed with PBS to remove the nonadherent cells.

Cell group 1 were stained by 3,6-bis(dimethylamino)acridine zinc chloride hydrochloride (Acridine Orange, Sigma, USA) and photographed under a fluorescence microscope (Nikon Ti-U, Japan).

Cell group 2 were fixed with $4 \%$ paraformaldehyde for $2 \mathrm{~h}$, stained by $4^{\prime}, 6^{\prime}$-diamidino-2-phenylindole (DAPI, Sigma, USA), then photographed under a fluorescence microscope and counted. All of the data were collected from at least three samples for each group and expressed as mean values \pm standard deviations. A value of $p<0.05$ was considered to be statistically significant.

Cell group 3 were fixed with $2.5 \%$ glutaraldehyde for $20 \mathrm{~min}$, stained with phalloidin-tetramethylrhodamine B isothiocyanate (phalloidin-TRITC, Sigma, USA) at room temperature in darkness for $1 \mathrm{~h}$ and further stained with DAPI for $5 \mathrm{~min}$, then photographed using laser confocal fluorescence microscopy (TCS SP5, Leica Microsystems, Germany).

Cell group 4 were fixed with $2.5 \%$ glutaraldehyde for $2 \mathrm{~h}$, and dehydrated in graded concentrations of ethanol $(30,50,70$, 90 , and $100 \%$ ). Afterward, the samples were dried in a freezedryer (Eyela FDU-1200, Tokyo Rikakikai, Japan), sputtered with a thin platinum layer, and then examined by SEM observations.

\section{Results and discussion}

\subsection{Fabrication of gradient micro-patterned OCP}

The fabrication of the superhydrophobic-superhydrophilic micropatterns on $\mathrm{TiO}_{2}$ NTA films using photocatalytic lithography is shown schematically in Fig. 1(A). A facile method was designed to fabricate gradient micro-patterned OCP coatings
A

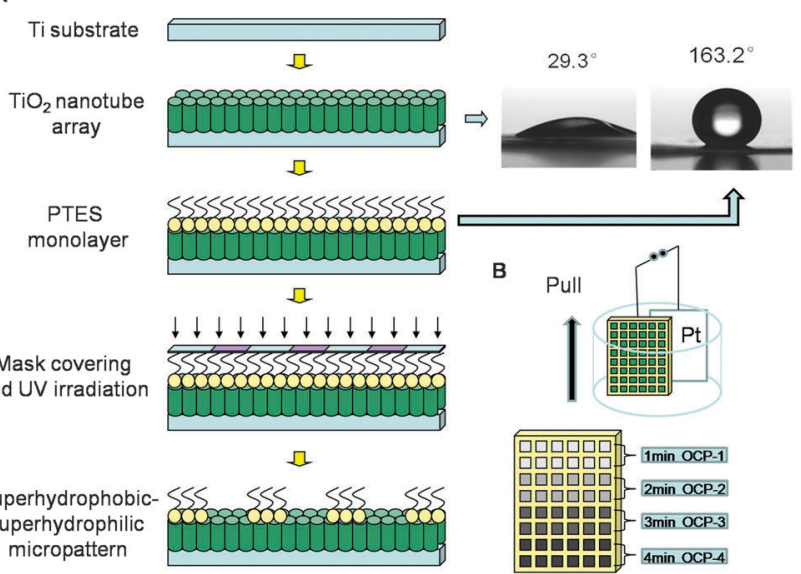

Fig. 1 Schematic outline of the preparation of superhydrophilicsuperhydrophobic micro-patterned template based on molecular selfassembly and photocatalytic lithography, with typical water contact angles on superhydrophilic and superhydrophobic surfaces shown in insets (A), and then fabrication of gradient OCP coatings on the micro-patterned $\mathrm{Ti}$ substrate by pull-stop-pull electrodeposition (B).

with biocompatible structure on medical titanium. Because of the extreme difference of wettability between the micro-units and the gaps on the superhydrophobic-superhydrophilic micropatterned template, the aqueous electrolyte contacted well and the OCP crystals were preferentially deposited on the superhydrophilic units, while the superhydrophobic locations with air trapped at the liquid/solid interface were prevented from contacting the electrolyte. Therefore, electrodeposition process can only take place in the superhydrophilic regions. Different deposition conditions such as deposition current density and deposition time can be controlled to fabricate gradient micropatterned OCP coatings with various morphologies and other structured $\mathrm{CaP}$ as well. Patterns with different shape and size can be realized by using various designs of photomasks.

The fabrication procedure of gradient OCP coatings on the micro-patterned Ti substrate is schematically shown in Fig. 1(B). The Ti sheets with superhydrophobic-superhydrophilic micropatterned template were immersed in aqueous electrolyte containing $0.042 \mathrm{~mol} \mathrm{~L}^{-1} \mathrm{Ca}\left(\mathrm{NO}_{3}\right)_{2}$ and $0.025 \mathrm{~mol} \mathrm{~L}^{-1} \mathrm{NH}_{4} \mathrm{H}_{2} \mathrm{PO}_{4}$ at $\mathrm{pH}$ 4.5. And a galvanostatic mode of $0.5 \mathrm{~mA} \mathrm{~cm}{ }^{-2}$ was supplied with a Pt sheet as counter electrode to deposit OCP film in the micro-patterns on the titanium surface for $1 \mathrm{~min}$. Then the sample was quickly pulled out from the solution for a distance of $2 \mathrm{~mm}$ while maintaining electrodeposition for another $1 \mathrm{~min}$, then again the sample was quickly pulled out from the solution for another distance of $2 \mathrm{~mm}$ while maintaining electrodeposition for another $1 \mathrm{~min}$. By such a multiple procedure of pull-stop-pull electrodeposition of OCP, the gradient micro-patterned OCP coatings were obtained as shown in Fig. 2, where the images of OCP-1, OCP-2, OCP-3 and OCP-4 indicated four locations of micro-patterned OCP on the same surface by electrodeposition for 1, 2, 3, and 4 min respectively. Therefore, the nucleation and growth of OCP crystals can be controlled on the different locations on the same surface to 


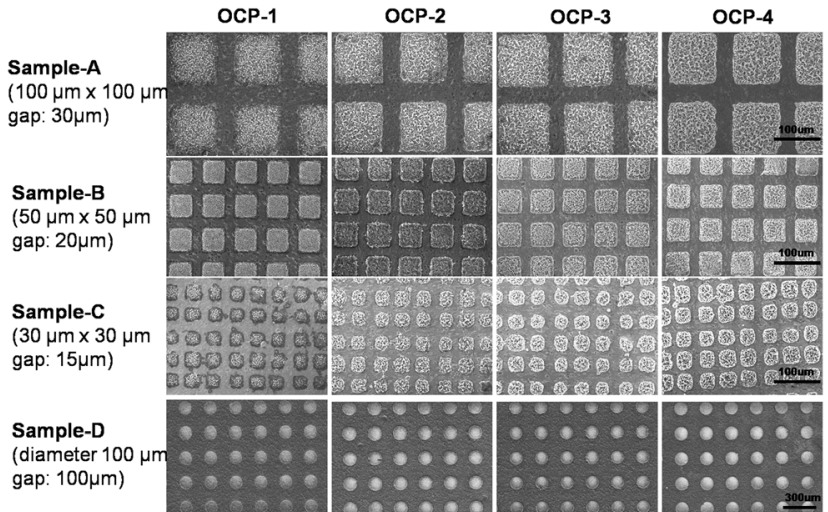

Fig. 2 SEM images of the gradient micro-patterned OCP coatings on superhydrophobic-superhydrophilic micro-patterned templates with different dimensions and structures for four samples (A-D): OCP-1 (1 min); OCP-2 (2 min); OCP-3 (3 min); OCP-4 (4 min).

finally form the gradient micro-patterned OCP coatings by adjusting of electrodeposition conditions such as deposition time and current density.

\subsection{Structure dependence of micro-patterned OCP on fabrication}

The 3D profile images of the gradient micro-patterned OCP coatings are exhibited in Fig. 3(A). The typical topography of the micro-patterned OCP coatings with nanostructure was orderly and uniform in three dimensions. There was an obvious increase in the thickness of the OCP coatings from OCP-1 to OCP-4 because of the increasing deposition time.

The EPMA images in Fig. 3(B) reflect the element distribution on micro-patterned OCP coatings visually. Elements of Ca

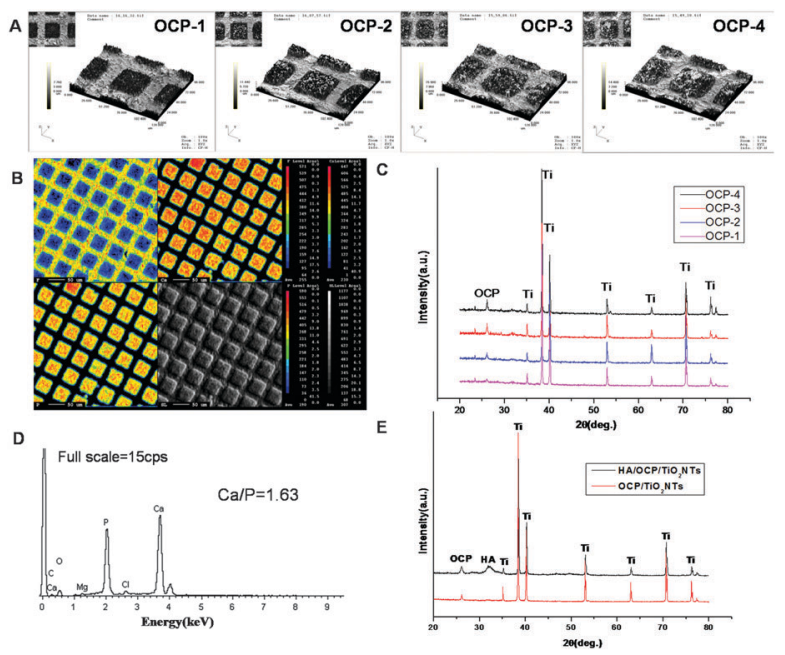

Fig. 3 3D profile images of the gradient micro-patterned OCP coatings, and the top-view images shown in inset (A); element distribution of the micro-patterned OCP coatings (B); XRD spectra of the gradient micropatterned OCP coatings (C); EDS spectrum of the bone-like apatite formed on the micro-patterned OCP coatings after soaking in SBF for 3 days (D); XRD spectra of the patterned OCP coatings and the micropatterned OCP coatings after soaking in SBF for 3 days (E). and $\mathrm{P}$ (corresponding to OCP) showed significantly large amounts in the superhydrophilic micro-units while $\mathrm{F}$ (corresponding to PTES) showed the opposite behavior. This indicated that almost complete removal and photodecomposition of fluoroalkyl chains took place in the micro-units under ultraviolet illumination, which led to the electrodeposition of OCP only at the superhydrophilic micro-units with distinct boundaries.

The XRD measurements shown in Fig. 3(C) confirm the presence of OCP with characteristic diffraction line of (002) plane at $26^{\circ}$ (JCPDS no. 44-778), while other reflections were ascribed to the $\mathrm{TiO}_{2} / \mathrm{Ti}$ substrate. As seen in the spectra, the intensity of the (002) peak of OCP was enhanced with increasing deposition time. The sharp diffraction peaks indicated a welldeveloped crystalline structure and a preferred crystal orientation with respect to the $c$-axis perpendicular to the substrate. The quantity of OCP crystals kept increasing with increasing deposition time since the intensity of an XRD peak was proportional to the quantity of a substance theoretically, which was also obviously displayed in the $3 \mathrm{D}$ profile images of the gradient micro-patterned OCP coatings.

\subsection{Evaluation of mineralization ability}

Fig. 4(A) shows the SEM images of the prepared gradient OCP coatings with different crystal morphologies changing from scattered-flower-like, scattered-flower-short-ribbon-like, shortribbon-like, to long-ribbon-like, due to the different time of electrodeposition. The SBF test was adapted to evaluate the mineralization ability and in vitro bioactivity of OCP coatings with various morphologies in the same physiological conditions. ${ }^{79}$ It was found that the different morphologies of OCP crystals showed significantly different ability of mineralization after 3 days soaking in SBF solution, as shown in Fig. 4(B). The scattered-flower-like morphology of the OCP crystals (OCP-1) was almost maintained while very few new $\mathrm{CaP}$ precipitates were visible on the surface of the original morphology of the OCP crystals (OCP-1), and much more CaP precipitates were observed on the scattered-flower-short-ribbon-like OCP crystals (OCP-2). Furthermore, the villus-like precipitates almost covered the short-ribbon-like and long-ribbon-like morphologies of the OCP crystals (OCP-3, OCP-4). The newly formed CaP layer on OCP coatings showed a similar morphology to that of natural bone. The EDS spectrum of the CaP precipitates formed

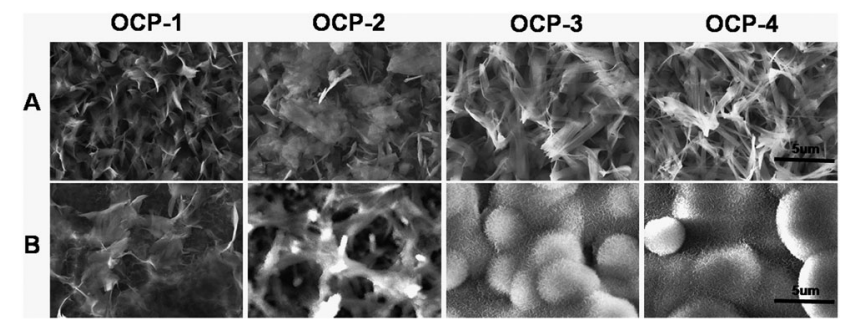

Fig. 4 SEM images of the gradient micro-patterned OCP coatings with different dimensions and structures: morphologies with a higher magnification of corresponding images of sample A in Fig. 2 (A); CaP precipitates on different locations of micro-patterned OCP coatings after soaking in SBF for 3 days (B). 
A $6 \mathrm{~h}$
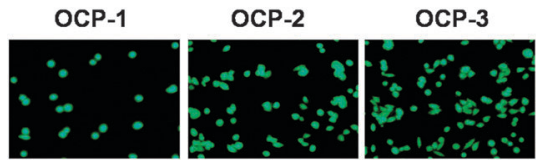

OCP-4
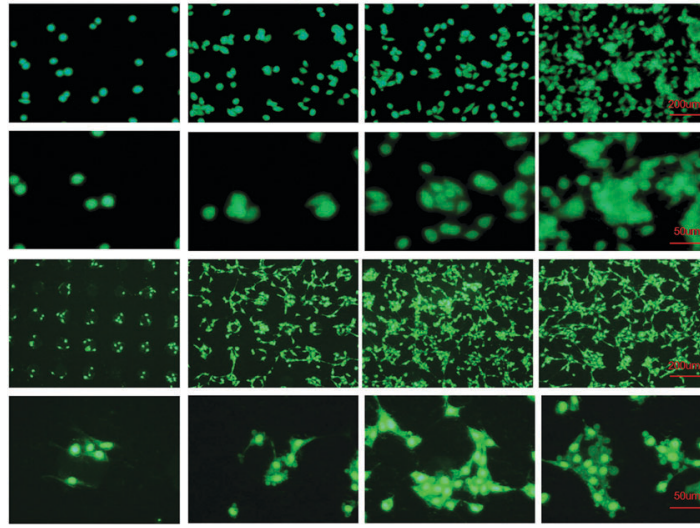

B

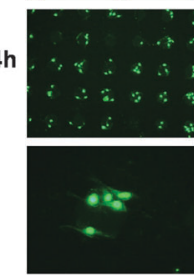

Fig. 5 Fluorescence images of MC3T3-E1 cells cultured on the gradient micro-patterned OCP coatings stained with Acridine Orange: $6 \mathrm{~h}$ (A); $24 \mathrm{~h}$ (B).

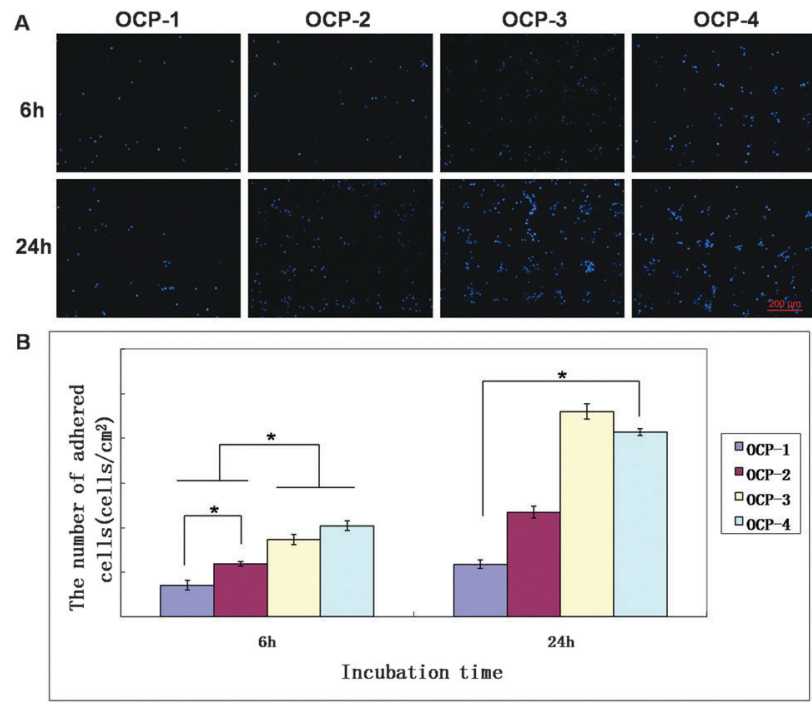

Fig. 6 Fluorescence images of MC3T3-E1 cells cultured on the gradient micro-patterned OCP coatings stained with DAPI after incubation for $6 \mathrm{~h}$ and $24 \mathrm{~h}(\mathrm{~A})$; the quantitative results of cell numbers. Significance: ${ }^{*} p<0.05$ (B).

on the micro-patterned OCP coatings after incubation in SBF for 3 days is shown in Fig. 3(D), indicating the presence of calcium and phosphorus as well as small amounts of sodium and magnesium. The $\mathrm{Ca} / \mathrm{P}$ atomic ratio of the precipitates newly formed was 1.63 , which was similar to that of HA phase. The XRD spectrum of the micro-patterned OCP coating after incubation in SBF for 3 days is shown in Fig. 3(E). After soaking in SBF for 3 days, the (002) reflection of OCP was still obtained and even dominant, indicating the crystal growth retained a preferred $c$-axis orientation during immersion in SBF. The mineral crystals in nature bone had a similar preferred orientation. Several small peaks appeared at $32-34^{\circ}$, which were consistent with the standard XRD peaks for HA (JCPDS no. 9-432). This implied that, after soaking in SBF for 3 days, the main phase on the OCP coatings evolved from OCP toward an apatite phase and the orientation of the newly formed apatite resulted from a similar structure of the prepared coatings. The significantly different mineralization behaviour of the gradient OCP coatings was demonstrated visually in the micro-patterns on the same surface of sample, which was an advanced evaluation with high efficiency and may avoid accidental error occurring among different samples and surroundings at the same time. The ability and bioactivity of OCP-4 was higher among the four, which proved that the surface composition, thickness and structure of the prepared coatings play a crucial role in the process of apatite formation in the body environment. ${ }^{80}$

\subsection{Assessment of cell responses}

The fluorescence images of MC3T3-E1 cells cultured on the same gradient micro-patterned OCP coatings stained by Acridine Orange and DAPI respectively are shown in Fig. 5 and 6(A), and the quantitative results of cell numbers are shown in Fig. 6(B). After $6 \mathrm{~h}$ incubation, MC3T3-E1 cells adhered almost onto the superhydrophilic micro-units and formed cell patterns on the OCP coatings, due to the different wettability between micro-units and gaps, and different biocompatibility of OCP micro-patterns as well. Moreover, some important proteins are involved in cellular adhesion and proliferation, such as fibronectin and vitronectin, which may preserve an active conformation on superhydrophilic regions rather than on superhydrophobic surfaces. ${ }^{81,82}$ The number of cells adhered onto the gradient OCP coatings was markedly different depending on the structures of OCP deposited for different times: only 1-2 cells in one unit of OCP-1 electrodeposited for $1 \mathrm{~min}$, 2-3 cells in one unit of OCP-2 prepared for $2 \mathrm{~min}, 2-6$ cells in one unit of OCP-3 precipitated for $3 \mathrm{~min}$, and 5-10 cells in one unit of OCP-4 deposited for $4 \mathrm{~min}$. According to the quantitative analysis shown in Fig. 6(B), the number of cells adhered on the micro-patterned units increased with the OCP thickness (prepared for different times) after $6 \mathrm{~h}$ culture. After $24 \mathrm{~h}$ incubation, the cells adhered onto the superhydrophilic microunits of OCP were well spread and began to proliferate. The number of cells adhered onto the different OCP coatings increased by $67 \%$ on OCP-1, 98\% on OCP-2, 166\% on OCP-3, and 103\% on OCP-4 respectively, compared to that incubated for $6 \mathrm{~h}$, indicating the cell activity on the different substrates was significantly different.

Cell adhesion, spreading activity and mobilization of cells attached on the different OCP units after $6 \mathrm{~h}$ and $24 \mathrm{~h}$ of incubation are assayed by staining with TRITC and DAPI to visualize the F-actin and nuclei respectively. ${ }^{83,84}$ The results are shown in Fig. 7, while Fig. 8 provides cell morphologies from SEM observations. It is indicated that the spreading of cells was remarkably regulated by the different OCP coatings during $6 \mathrm{~h}$ incubation. The cells adhered with a spherical or fusiform shape onto the micro-units of OCP-1, less bioactive because of being too thin for OCP coatings. And the cells spread out with a fusiform shape on OCP-2, and a small amount of filopodia was stretched out. In contrast, the cells on the OCP-3 micro-units displayed a polygonal shape, while cells on OCP-4 spread much better with a polygonal shape and prominent filopodia and lamellipodia protrusions. ${ }^{85,86}$ The expression of F-actin on OCP-4 was significantly better than that on OCP-1, OCP-2 and OCP-3. After $24 \mathrm{~h}$ 
A

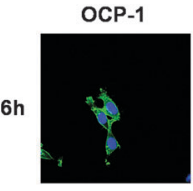

B
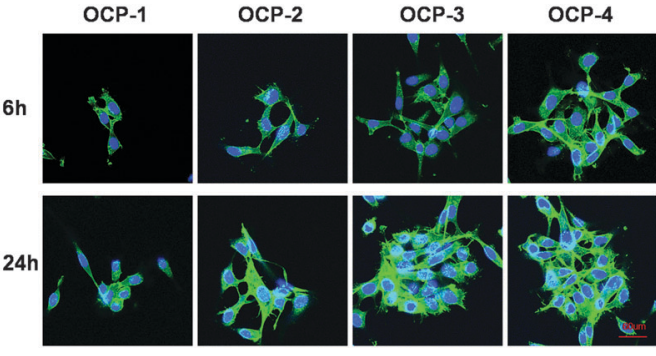

Fig. 7 Fluorescence images of MC3T3-E1 cells cultured on the gradient micro-patterned OCP coatings. Cytoskeletal actin staining with phalloidinTRITC (green) and nuclei staining with DAPI (blue): $6 \mathrm{~h}$ (A); $24 \mathrm{~h}$ (B).

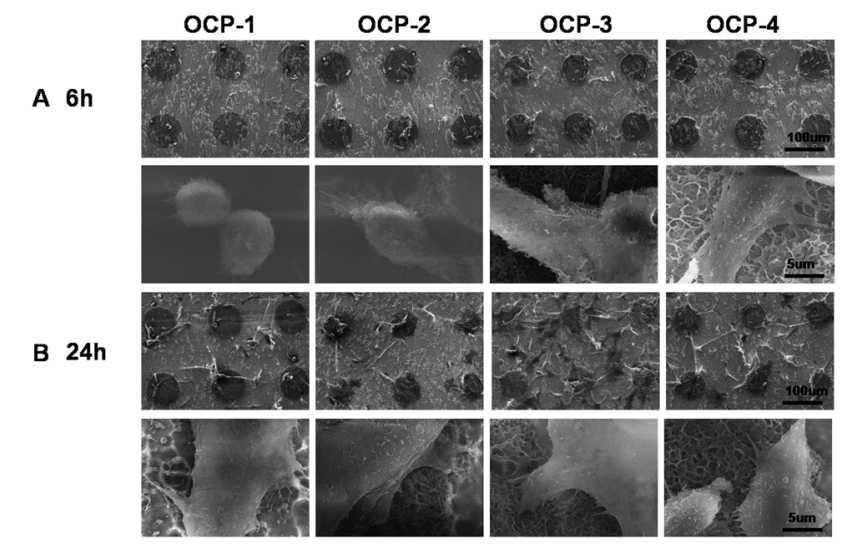

Fig. 8 SEM images of MC3T3-E1 cells cultured on the gradient micropatterned OCP coatings: $6 \mathrm{~h}(\mathrm{~A}) ; 24 \mathrm{~h} \mathrm{(B)}$.

incubation, most cells were well spread and began to proliferate, with a long fusiform shape on the micro-units with OCP-1 and with a polygonal shape on OCP-2, most cell bodies on the microunits and some prominent filopodia on the superhydrophobic gaps. Apart from the difference in the number of cells adhered onto the different OCP coatings, the spread of cells behaved remarkably different, implying that the different bioactivity closely depended on the structures of OCP coatings in the micro-units. Cells spread out crowdedly and actively on the micro-units with OCP-3 and much more obviously on OCP-4 with extended polygonal shape and a large amount of prominent filopodia and lamellipodia protrusions, cell podia bridging over in the single micro-units and among different micro-units. A few cells adhered onto the superhydrophobic gaps with low viability because of the space limitation. The expression of F-actin on OCP-2, OCP-3 and OCP-4 was better than on OCP-1. Mobilization of cells can be indicated in the fluorescence and SEM images, too. For OCP micro-units with gradient coatings, cells tended to adhere to the border of the micro-units in the initial adhesion and spread out to the inner part of the micro-units until the units were fully occupied and then had to spread out of the micro-units.

During the initial adhesion, the coatings of OCP-4 showed the best bioactivity with most cell adhesion and active cell spreading. It was found, after incubation for 24 hours, that the coatings of OCP-3 were able to modulate the cells to be more stereoscopic with the fastest proliferation, indicating a superior bioactivity comparing with OCP coatings in micro-units. As a result, the composition, thickness and structure of the OCP coatings play a crucial role in the adhesion, spreading, and proliferation of mouse MC3T3-E1 cells.

All in vitro tests of MC3T3-E1 cells demonstrated that the cells showed different numbers and morphology adhered on the different micro-units in which cells were effectively limited, indicating that the cells are highly sensitive to the gradient structure of OCP coatings, and the gradient micro-patterns of biomaterials can be a powerful tool for not only highthroughput evaluation of biocompatibility and bioactivity, but also highly efficient design and construction of various advanced biosurfaces.

\section{Conclusions}

In summary, we have successfully developed an approach for fabricating biocompatible gradient micro-patterned OCP coatings based on a superhydrophobic-superhydrophilic micropatterned template. The patterned OCP coatings with different morphologies showed significantly different ability of mineralization in SBF and bioactivity in the adhesion, spreading and proliferation of mouse MC3T3-E1 cells. The equipment we used in our approach is very simple and can be easily used in general laboratory conditions. In our approach, the substrate material can be titanium or other metallic materials, and various biomaterials such as calcium phosphate, proteins, antibacterial agents etc. can be deposited to the superhydrophilicsuperhydrophobic micro-patterned templates by wet chemical methods to fabricate functional gradient micro-patterned coatings. The gradient micro-patterned OCP coatings described in this paper are just an example of the construction of gradient micro-patterned coatings. Because titanium is a widely used biomaterial and also electrical conductor, the electrochemical method can be used to deposit various biofilms on the superhydrophilic-superhydrophobic micro-patterned templates in more accurate and controllable mode. It can become a facile and efficient technique for fabrication of various gradient and micro-patterned functional materials by using the strategy of superhydrophobic-superhydrophilic micro-patterned template and pull-stop-pull approach. Meanwhile, the bioactive micropatterns with gradient coatings are an effective modulation for spatial control of dynamic cell attachment and proliferation, which enables high-throughput evaluation of cell behaviors, interaction of cells and materials, and bioproperties in a single experiment. The development of gradient micro-patterns is promising for not only high-throughput evaluation of biocompatibility and bioactivity, but also highly efficient design and construction of various functional materials.

\section{Acknowledgements}

The authors are grateful for the financial support from the Natural Science Foundation of China (51571169 and 21321062) and International Cooperation Program of China (2014DFG52350). 


\section{Notes and references}

1 J. Y. Wong, J. B. Leach and X. Q. Brown, Surf. Sci., 2004, 570, 119-133.

2 C. Chai and K. W. Leong, Mol. Ther., 2007, 15, 467-480.

3 D. E. Discher, P. Janmey and Y. L. Wang, Science, 2005, 310, 1139-1143.

4 D. Guarnieri, A. De Capua, M. Ventre, A. Borzacchiello, C. Pedone, D. Marasco, M. Ruvo and P. A. Netti, Acta Biomater., 2010, 6, 2532-2539.

5 P. Costa, J. E. Gautrot and J. T. Connelly, Acta Biomater, 2014, 10, 2415-2422.

6 B. Cao, Z. H. Li, R. Peng and J. D. Ding, Biomaterials, 2015, 64, 21-32.

7 F. Ye, B. H. Ma, J. Gao, L. Xie, C. Wei and J. Jiang, J. Biomed. Mater. Res., Part B, 2015, 103, 1375-1380.

8 M. Thery, J. Cell Sci., 2010, 123, 4201-4213.

9 Y. Nahmias and D. J. Odde, Nat. Protoc., 2006, 1, 2288-2296.

10 M. S. Hahn, L. J. Taite, J. J. Moon, M. C. Rowland, K. A. Ruffino and J. L. West, Biomaterials, 2006, 27, 2519-2524.

11 Y. He, X. Wang, L. Chen and J. D. Ding, J. Mater. Chem. B, 2014, 2, 2220-2227.

12 X. Zhang, F. Shi, J. Niu, Y. G. Jiang and Z. Q. Wang, J. Mater. Chem., 2008, 18, 621-633.

13 X. J. Feng and L. Jiang, Adv. Mater., 2006, 18, 3063-3078.

14 L. Marcon, A. Addad, Y. Coffinier and R. Boukherroub, Acta Biomater., 2013, 9, 4585-4591.

15 G. Jing, Y. Wang, T. Zhou, S. F. Perry, M. T. Grimes and S. Tatic-Lucic, Acta Biomater., 2011, 7, 1094-1103.

16 Y. H. Lai, J. T. Yang and D. B. Shieh, Lab Chip, 2010, 10, 499-504.

17 K. Hecht, N. Schuler, A. Dubbe, M. Kraut, P. Pfeifer and R. Dittmeyer, J. Chem. Eng. Jpn., 2012, 45, 727-733.

18 L. Zhang, H. Z. Yu, N. Zhao, Z. M. Dang and J. Xu, J. Appl. Polym. Sci., 2014, 131, 6.

19 Q. M. Yin, J. M. Ye and Y. L. Zhou, Chem. J. Chin. Univ.-Chin., 2008, 29, 1647-1649.

20 J. El-Ali, P. K. Sorger and K. F. Jensen, Nature, 2006, 442, 403-411.

21 X. Y. Jiang, D. A. Bruzewicz, A. P. Wong, M. Piel and G. M. Whitesides, Proc. Natl. Acad. Sci. U. S. A., 2005, 102, 975-978.

22 R. Kapur, K. A. Giuliano, M. Campana, T. Adams, K. Olson, D. Jung, M. Mrksich, C. Vasudevan and D. L. Taylor, Biomed. Microdevices, 1999, 2, 99-109.

23 J. Yang, M. Yamato and T. Okano, MRS Bull., 2005, 30, 189-193.

24 N. M. Alves, J. Shi, E. Oramas, J. L. Santos, H. Tomas and J. F. Mano, J. Biomed. Mater. Res., Part A, 2009, 91A, 480-488.

25 C. Yan, J. G. Sun and J. D. Ding, Biomaterials, 2011, 32, 3931-3938.

26 I. Y. Wong, B. D. Almquist and N. A. Melosh, Mater. Today, 2010, 13, 14-22.

27 C. A. Goubko and X. D. Cao, Mater. Sci. Eng., C, 2009, 29, 1855-1868.

28 J. Shi, N. M. Alves and J. F. Mano, Bioinspiration Biomimetics, 2008, 3, 034003.
29 P. E. Scopelliti, A. Borgonovo, M. Indrieri, L. Giorgetti, G. Bongiorno, R. Carbone, A. Podesta and P. Milani, PLoS One, 2010, 5, e11862.

30 Y. Ito and M. Nogawa, Biomaterials, 2003, 24, 3021-3026.

31 D. M. Thompson and H. M. Buettner, Tissue Eng., 2001, 7, 247-265.

32 J. Tang, R. Peng and J. D. Ding, Biomaterials, 2010, 31, 2470-2476.

33 X. T. Shi, L. Li, S. Ostrovidov, Y. W. Shu, A. Khademhosseini and H. K. Wu, ACS Appl. Mater. Interfaces, 2014, 6, 11915-11923.

34 F. Yao, H. Hu, S. L. Xu, R. J. Huo, Z. P. Zhao, F. Z. Zhang and F. J. Xu, ACS Appl. Mater. Interfaces, 2015, 7, 3882-3887.

35 R. Branemark, L. O. Ohrnell, R. Skalak, L. Carlsson and P. I. Branemark, J. Orthop. Res., 1998, 16, 61-69.

36 J. E. Davies, Anat. Rec., 1996, 245, 426-445.

37 M. Ysander, R. Branemark, K. Olmarker and R. R. Myers, J. Rehab. Res. Dev., 2001, 38, 183-190.

38 J. E. Davies and N. Baldan, J. Biomed. Mater. Res., 1997, 36, 429-440.

39 W. J. Weng, S. Zhang, K. Cheng, H. B. Qu, P. Y. Du, G. Shen, J. Yuan and G. R. Han, Surf. Coat. Technol., 2003, 167, 292-296.

40 W. Pompe, H. Worch, W. Habraken, P. Simon, R. Kniep, H. Ehrlich and P. Paufler, J. Mater. Chem. B, 2015, 3, 5318-5329.

41 D. Sriranganathan, N. Kanwal, K. A. Hing and R. G. Hill, J. Mater. Sci.: Mater. Med., 2016, 27, DOI: 10.1007/s10856015-5653-6.

42 W. E. Brown, N. Eidelman and B. Tomazic, Adv. Dent. Res., 1987, 1, 306-313.

43 M. S. A. Johnsson and G. H. Nancollas, Crit. Rev. Oral Biol. Med., 1992, 3, 61-82.

44 P. L. Jiang, J. H. Liang, R. Song, Y. M. Zhang, L. Ren, L. H. Zhang, P. F. Tang and C. J. Lin, ACS Appl. Mater. Interfaces, 2015, 7, 14384-14396.

45 Z. Hadisi, J. Nourmohammadi and J. Mohammadi, Ceram. Int., 2015, 41, 10745-10754.

46 Y. Liu, R. M. Shelton, U. Gbureck and J. E. Barralet, J. Biomed. Mater. Res., Part A, 2009, 90A, 972-980.

47 M. Yamaguchi, K. Ikeda, M. Suzuki, A. Kiyohara, S. N. Kudoh, K. Shimizu, T. Taira, D. Ito, T. Uchida and K. Gohara, Langmuir, 2011, 27, 12521-12532.

48 A. Shishido, I. B. Diviliansky, I. C. Khoo, T. S. Mayer, S. Nishimura, G. L. Egan and T. E. Mallouk, Appl. Phys. Lett., 2001, 79, 3332-3334.

49 J. B. Gan, H. Chen, F. Zhou, H. Huang, J. Zheng, W. Song, L. Yuan and Z. K. Wu, Colloids Surf., B, 2010, 76, 381-385.

50 J. Doh and D. J. Irvine, J. Am. Chem. Soc., 2004, 126, 9170-9171.

51 A. Scandurra, G. F. Indelli and S. Pignataro, Surf. Interface Anal., 2012, 44, 1171-1176.

52 X. M. Zhao, Y. N. Xia and G. M. Whitesides, J. Mater. Chem., 1997, 7, 1069-1074.

53 S. H. Park and Y. N. Xia, Chem. Mater., 1998, 10, 1745-1747. 54 J. Peng, Y. C. Han, J. Fu, Y. M. Yang and B. Y. Li, Macromol. Chem. Phys., 2003, 204, 125-130. 
55 E. Ostuni, C. S. Chen, D. E. Ingber and G. M. Whitesides, Langmuir, 2001, 17, 2828-2834.

56 N. Tanaka, H. Ota, K. Fukumori, J. Miyake, M. Yamato and T. Okano, Biomaterials, 2014, 35, 9802-9810.

57 L. Filipponi, P. Livingston, O. Kaspar, V. Tokarova and D. V. Nicolau, Biomed. Microdevices, 2016, 18, DOI: 10.1007/s10544-016-0036-4.

58 P. Xiao, J. C. Gu, J. Chen, J. W. Zhang, R. B. Xing, Y. C. Han, J. Fu, W. Q. Wang and T. Chen, Chem. Commun., 2014, 50, 7103-7106.

59 S. Han, S. Hong, H. W. Kang, M. Wanit, B. Kang and S. H. Ko, J. Micromech. Microeng., 2015, 25, 105006.

60 M. J. Jang and Y. Nam, Macromol. Biosci., 2015, 15, 613-621.

61 K. B. Kim, M. S. Kim, D. H. Lee, B. M. Choi, K. S. Jung, S. H. Jung, J. K. Lee, O. Beom-Hoan, S. G. Lee and S. G. Park, Microelectron. Eng., 2015, 145, 160-165.

62 S. R. Sousa, P. Moradas-Ferreira, B. Saramago, L. V. Melo and M. A. Barbosa, Langmuir, 2004, 20, 9745-9754.

63 W. Han, Y. D. Wang and Y. F. Zheng, in Multi-Functional Materials and Structures, Pts 1 and 2, ed. A. K. T. Lau, J. Lu, V. K. Varadan, F. K. Chang, J. P. Tu and P. M. Lam, 2008, vol. 47-50, pp. 1438-1441.

64 D. Chen, Y. F. Gao, G. Wang, H. Zhang, W. Lu and J. H. Li, J. Phys. Chem. C, 2007, 111, 13163-13169.

65 O. Carp, C. L. Huisman and A. Reller, Prog. Solid State Chem., 2004, 32, 33-177.

66 A. Fujishima, X. T. Zhang and D. A. Tryk, Surf. Sci. Rep., 2008, 63, 515-582.

67 H. Noh, S. G. Oh and S. S. Im, Appl. Surf. Sci., 2015, 333, 157-162.

68 M. K. Nazeeruddin, P. Pechy, T. Renouard, S. M. Zakeeruddin, R. Humphry-Baker, P. Comte, P. Liska, L. Cevey, E. Costa, V. Shklover, L. Spiccia, G. B. Deacon, C. A. Bignozzi and M. Gratzel, J. Am. Chem. Soc., 2001, 123, 1613-1624.

69 J. Y. Lee, C. Jones, M. A. Zern and A. Revzin, Anal. Chem., 2006, 78, 8305-8312.
70 R. Zhang, M. Chen, Q. J. Xue, F. Guan and S. Liang, Chem. J. Chin. Univ.-Chin., 2005, 26, 939-941.

71 K. Nakata, S. Nishimoto, A. Kubo, D. Tryk, T. Ochiai, T. Murakami and A. Fujishima, Chem. - Asian J., 2009, 4, 984-988.

72 J. T. Park, D. K. Roh, R. Patel, K. J. Son, W. G. Koh and J. H. Kim, Electrochim. Acta, 2010, 56, 68-73.

73 Y. K. Lai, Z. Q. Lin, Z. Chen, J. Y. Huang and C. J. Lin, Mater. Lett., 2010, 64, 1309-1312.

74 F. Guan, M. Chen, R. Zhang, S. Liang and Q. J. Xue, Chem. J. Chin. Univ.-Chin., 2005, 26, 599-602.

75 S. Liang, M. Chen, Q. J. Xue, Y. L. Qi and J. M. Chen, J. Colloid Interface Sci., 2007, 311, 194-202.

76 G. K. Mor, O. K. Varghese, M. Paulose and C. A. Grimes, Adv. Funct. Mater., 2005, 15, 1291-1296.

77 S. Berger, A. Ghicov, Y. C. Nah and P. Schmuki, Langmuir, 2009, 25, 4841-4844.

78 Y. K. Lai, J. Y. Huang, J. J. Gong, Y. X. Huang, C. L. Wang, Z. Chen and C. J. Lin, J. Electrochem. Soc., 2009, 156, D480-D484.

79 S. Hiromoto, M. Inoue, T. Taguchi, M. Yamane and N. Ohtsu, Acta Biomater., 2015, 11, 520-530.

80 J. Kunze, L. Muller, J. M. Macak, P. Greil, P. Schmuki and F. A. Muller, Electrochim. Acta, 2008, 53, 6995-7003.

81 T. Ishizaki, N. Saito and O. Takai, Langmuir, 2010, 26, 8147-8154.

82 D. J. Iuliano, S. S. Saavedra and G. A. Truskey, J. Biomed. Mater. Res., 1993, 27, 1103-1113.

83 J. S. Choi, D. H. Kim and T. S. Seo, Biomaterials, 2016, 84, 315-322.

84 L. C. Boraas, J. B. Guidry, E. T. Pineda and T. Ahsan, PLoS One, 2016, 11, DOI: 10.1371/journal.pone.0145084.

85 S. Lumetti, E. Manfredi, S. Ferraris, S. Spriano, G. Passeri, G. Ghiacci, G. Macaluso and C. Galli, J. Mater. Sci.: Mater. Med., 2016, 27, 9.

86 H. S. Park, S. Y. Lee, H. Yoon and I. Noh, Pure Appl. Chem., 2014, 86, 1911-1922. 